University of South Florida

DIGITAL COMMONS

Digital Commons @ University of

@ UNIVERSITY OF SOUTH FLORIDA

South Florida

$1-1-2011$

\title{
2011 Annual Report USF Sarasota-Manatee
}

USF

Follow this and additional works at: https://digitalcommons.usf.edu/usf_accountability_reports

\section{Scholar Commons Citation}

USF, "2011 Annual Report USF Sarasota-Manatee" (2011). USF Accountability Reports. 17.

https://digitalcommons.usf.edu/usf_accountability_reports/17

This Article is brought to you for free and open access by the USF Archives at Digital Commons @ University of South Florida. It has been accepted for inclusion in USF Accountability Reports by an authorized administrator of Digital Commons @ University of South Florida. For more information, please contact digitalcommons@usf.edu. 


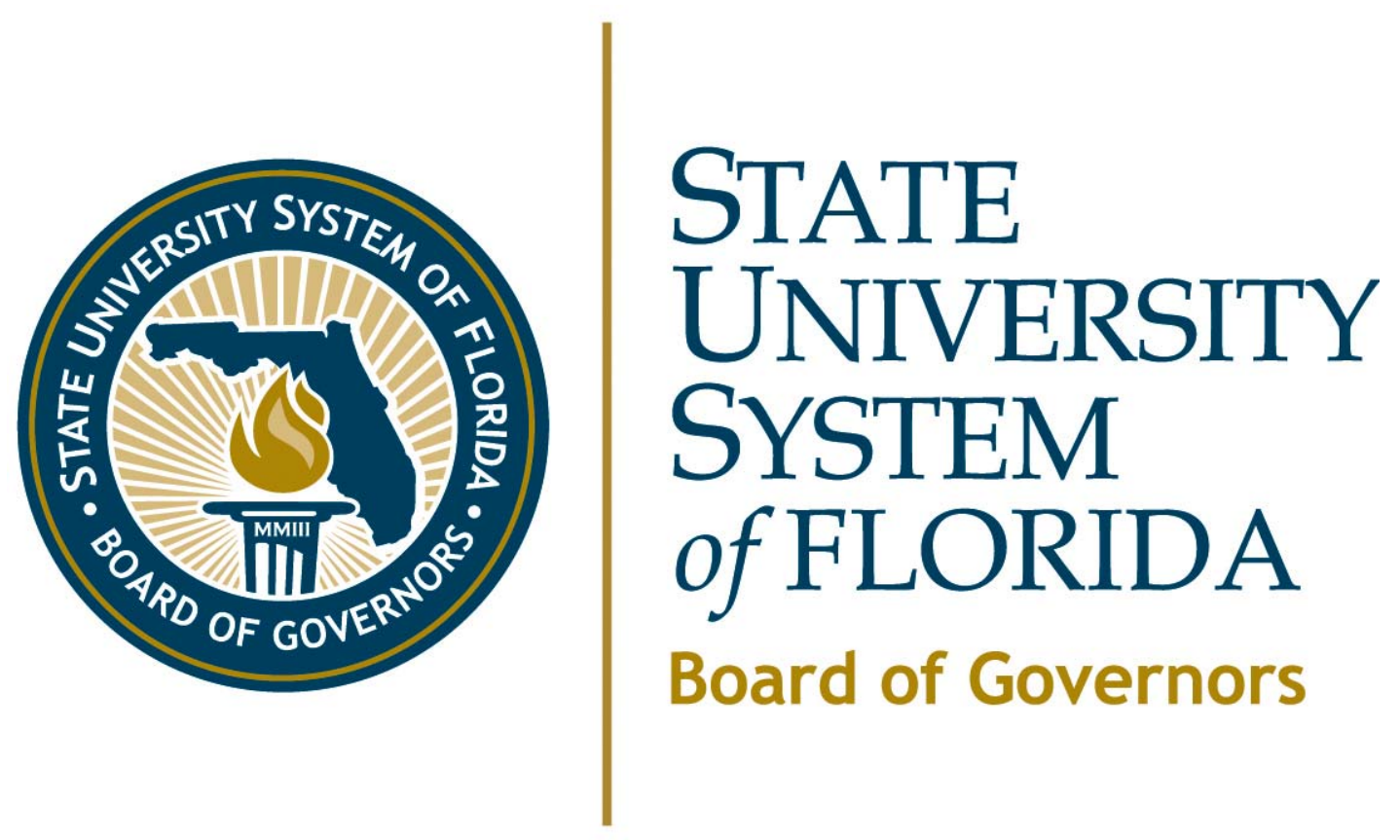

University of South Florida - Sarasota-Manatee 2010-11 Annual Report 
Data definitions are provided in the Appendices.

\footnotetext{
Note concerning data accuracy: The Office of the Board of Governors believes that the accuracy of the data it collects and reports is paramount to ensuring accountability in the State University System. Thus, the Board Office allows university resubmissions of some data to correct errors when they are discovered. This policy can lead to changes in historical data.
} 


\section{TABLE OF CONTENTS}

Dashboard. page 1

Key Achievements page 4

Narrative Report page 5

Data Tables page 9

\section{Section 1 - Financial Resources}

[Not every university will have HSC \& IFAS tables.]

TABLE 1A. University Education and General Revenues

1B. University Education and General Expenditures

1C. State Funding per Full-Time Equivalent (FTE) Student

1D. University Other Budget Entities

1E. University Total Revenues and Expenditures

1F. Voluntary Support of Higher Education

1G. University Federal Stimulus Dollars (ARRA)

1H. HSC - Education and General Revenues

1I. HSC - Education and General Expenditures

1J. HSC - Faculty Practice Plans

1K. IFAS - Education and General Revenues

1L. IFAS - Education and General Expenditures

\section{Section 2 - Personnel}

TABLE 2A. Personnel Headcount

\section{Section 3 - Enrollment}

TABLE 3A. University Full-Time Enrollment (FTE)

3B. Enrollment by Location

\section{Section 4 - Undergraduate Education}

TABLE 4A. Baccalaureate Degree Program Changes in AY 2009-2010

4B. First-Year Persistence Rates

4C. Federal Definition - Undergraduate Progression and Graduation Rates for Full-Time,

First-Time-in-College (FTIC) Students
Section 4 - Undergraduate Education (continued)

TABLE 4D. SUS Definition - Undergraduate Progression and Graduation Rates for Full- \& Part-Time, First-Time-in-College (FTIC) Students

4E. SUS Definition - Undergraduate Progression and Graduation Rates for AA Transfer Students

4F. SUS Definition - Undergraduate Progression and Graduation Rates for Other Transfer Students

4G. Baccalaureate Degrees Awarded

4H. Baccalaureate Degrees Awarded in Areas of Strategic Emphasis

4I. Baccalaureate Degrees Awarded to Underrepresented Groups

4J. Baccalaureate Completion Without Excess Credit Hours

4K. Undergraduate Course Offerings

4L. Faculty Teaching Undergraduates

4M. Undergraduate Instructional Faculty Compensation

4N. Student/Faculty Ratio

4O. Professional Licensure Exams - Undergraduate Programs

4P. Tuition Differential Fee

\section{Section 5 - Graduate Education}

TABLE 5A. Graduate Degree Program Changes in AY 2009-2010

5B. Graduate Degrees Awarded

5C. Graduate Degrees Awarded in Areas of Strategic Emphasis

5D. Professional Licensure Exams - Graduate Programs

\section{Section 6 - Research and Economic Development}

TABLE 6A. Research and Development

6B. Centers of Excellence

6C. State University Research Commercialization Assistance Grants

6D. 21st Century World Class Scholars Program 


\begin{tabular}{|c|c|c|c|c|c|c|c|}
\hline \multicolumn{8}{|c|}{ University of South Florida - Sarasota-Manatee 2011 Annual Report } \\
\hline \multicolumn{3}{|c|}{ Sites and Campuses } & \multicolumn{5}{|c|}{ USF Sarasota-Manatee Campus } \\
\hline Enrollments & Headcount & $\%$ & \multicolumn{3}{|c|}{ Degree Programs Offered (As of Spr. 2011) } & \multicolumn{2}{|c|}{ Carnegie Classification } \\
\hline $\begin{array}{c}\text { TOTAL } \\
\text { (Fall 2010) }\end{array}$ & 1,897 & $100 \%$ & \multicolumn{2}{|l|}{ TOTAL } & \multirow{5}{*}{$\begin{array}{l}\text { Total } \\
\text { programs } \\
\text { reflected } \\
\text { at the } \\
\text { USF } \\
\text { System } \\
\text { level. }\end{array}$} & $\begin{array}{c}\text { Undergraduate } \\
\text { Instructional Program: }\end{array}$ & $\begin{array}{c}\text { Prof+A\&S/SGC: Professions plus arts \& sciences, } \\
\text { some graduate coexistence }\end{array}$ \\
\hline Black & 113 & $6 \%$ & Baccalaureat & & & Graduate Instructional & Postbac-A\&S/Ed: Postbaccalaureate with arts \& \\
\hline Hispanic & 178 & $9 \%$ & Master's \& Speci & ist's & & Program: & sciences (education dominant) \\
\hline White & 1,474 & $78 \%$ & Research Docto & & & Enrollment Profile: & HU: High undergraduate \\
\hline Other & 132 & $7 \%$ & \multicolumn{2}{|c|}{ Professional Doctorate } & & Undergraduate Profile: & PT4: Higher part-time four-year \\
\hline Full-Time & 831 & $44 \%$ & \multirow{2}{*}{ Faculty (Fall 2010) } & \multirow{2}{*}{$\begin{array}{l}\text { Full- } \\
\text { Time }\end{array}$} & \multirow{2}{*}{$\begin{array}{l}\text { Part- } \\
\text { Time }\end{array}$} & Size and Setting: & S4/NR: Small four-year, primarily nonresidential \\
\hline Part-Time & 1,066 & $56 \%$ & & & & \multirow{2}{*}{ Basic: } & \multirow{2}{*}{$\begin{array}{l}\text { Master's M: Master's Colleges and Universities } \\
\text { (medium programs) }\end{array}$} \\
\hline Undergraduate & 1,533 & $81 \%$ & TOTAL & 49 & 87 & & \\
\hline Graduate & 184 & $10 \%$ & Tenure/T. Track & 32 & 1 & \multirow{2}{*}{ Elective Classification: } & \multirow{2}{*}{ N/A } \\
\hline Unclassified & 180 & $9 \%$ & Other Faculty/Instr. & 17 & 86 & & \\
\hline
\end{tabular}

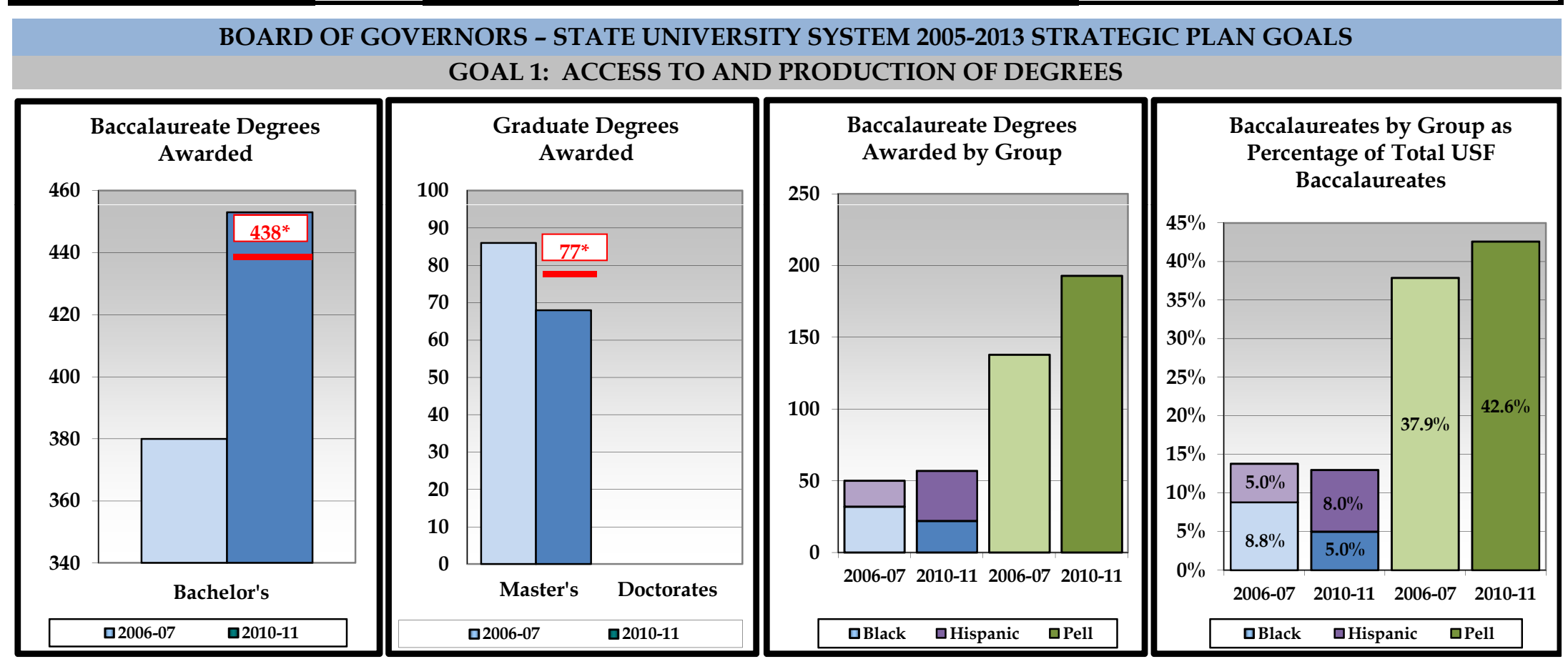




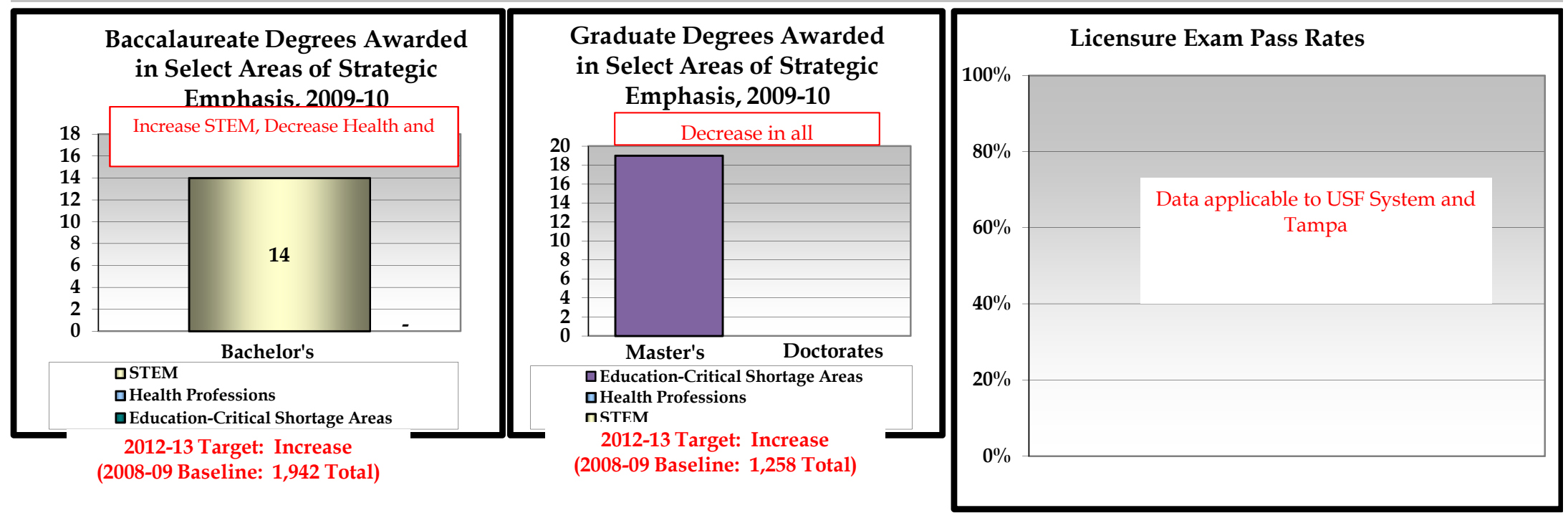

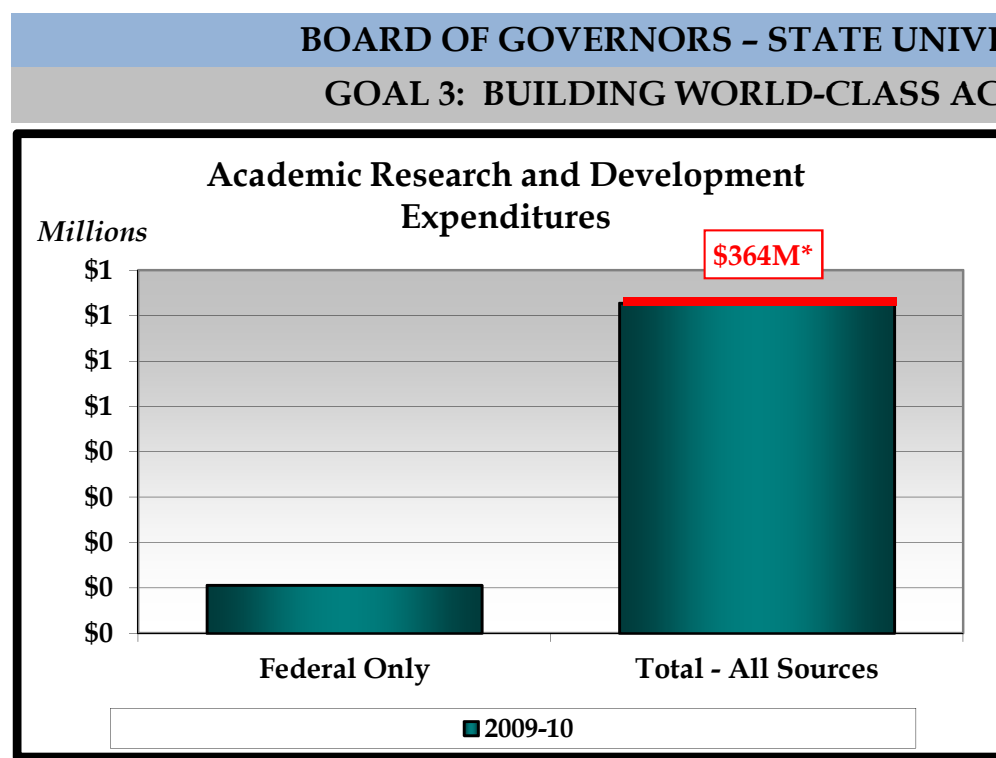

*2011-12 Targets for Research \& Development Expenditures.

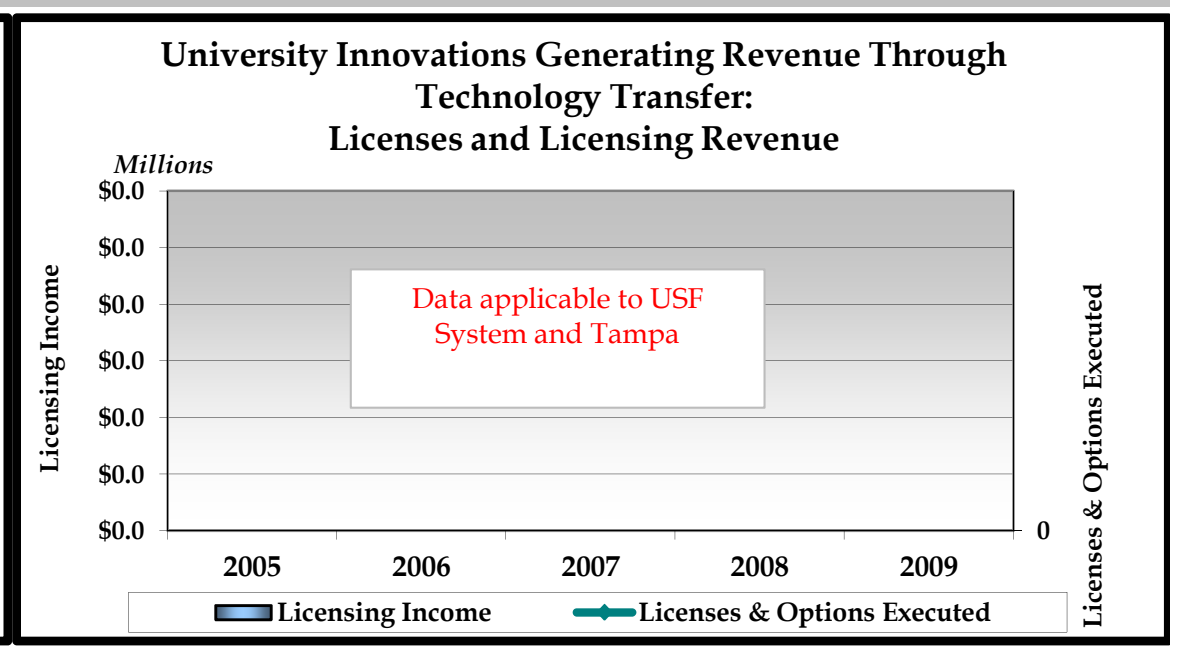

2011-12 Targets: Licenses - Increase (2008 Baseline $=23)$ Licensing Revenue - Increase (2008 Baseline $=\$ 2,099,712)$ 
RESOURCES, EFFICIENCIES, AND EFFECTIVENESS

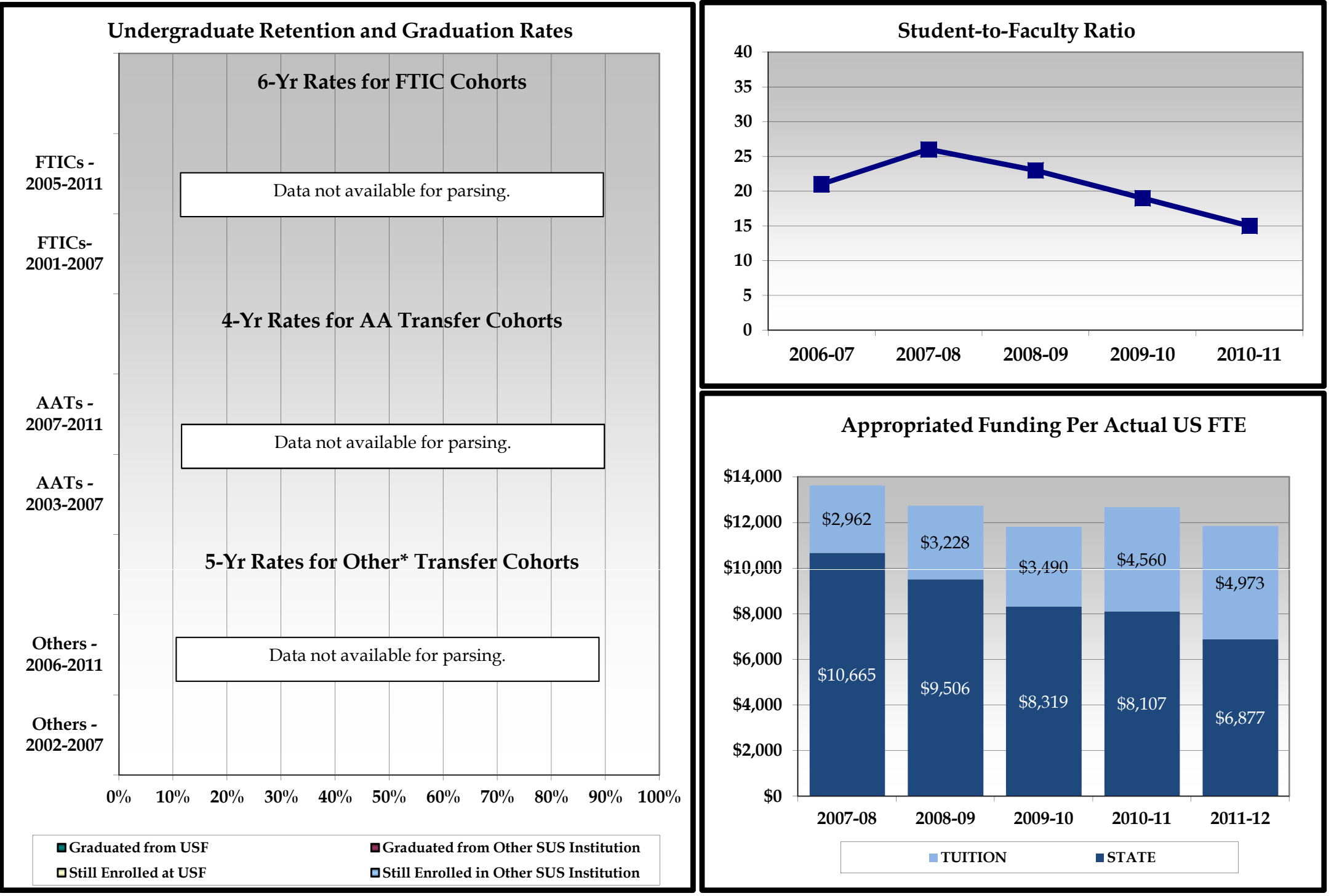

* The composition of "Other Transfer" cohorts may vary greatly by institution and by year.

TUITION is the appropriated budget authority, not the amount actually collected. Does not include non-instructional local fees.

**Graduation Rate from SAME Institution.

STATE includes General Revenues, Lottery and Other Trust funds (ie. Federal Stimulus for 2009-10 and 2010-11 only). 


\section{- Student awards/achievements}

1. Information Technology major awarded a Science, Mathematics and Research for Transformation (SMART) Scholarship from the United States Department of Defense.

2. Elementary Education major awarded Florida Commission on the Status of Women Achievement Award for her outstanding contributions and service to her community.

\section{- Faculty awards/achievements}

1. USFSM professor Dr. Thomas Crisp received the Marguerite Cogorno Radencich Award honoring Florida's Outstanding Teacher Educator in Reading.

\section{- Program awards/achievements}

1. USFSM College of Education sponsored the 2011 Annual Children's Literature Symposium - a joint conference with West Central Region F.A.T.E.

\section{- Research awards/achievements}

1. A College of Arts \& Sciences professor received a $\$ 131,566$ sub-contract from SCOPE to conduct research for The Aging with Dignity and Independence Initiative.
2. The article published by two College of Education professors in the Journal of Intergenerational Relationships (JIR): “Lessons from Erikson: A look at autonomy across lifespan" has been cited more than any other in the journal. (202 times)

3. A College of Education professor was awarded as a Co-PI a grant from the Social Science Research and Humanities Council of Canada to research children affected by war in northern Uganda. She will be traveling to Uganda in November.

\section{- Institutional awards/achievements}

1. USF Sarasota-Manatee was granted separate accreditation from the Southern Association of Colleges and Schools (SACS) in 2011.

2. Fall 2010, USFSM opened its new USFSM @ North Port instructional site. The facility allows USFSM to provide access to baccalaureate and master's programs to students living in south Sarasota County.

3. USF Sarasota-Manatee received a one-year, $\$ 247,500$ grant from the Health Resources and Services Administration (HRSA). To date 


\section{USF - SARASOTA-MANATEE \\ 2010-11 NARRATIVE REPORT}

\section{INTRODUCTION}

\section{Mission}

The University of South Florida Sarasota-Manatee delivers quality education to upper-division baccalaureate and master's students in an active research environment. We support students in attaining their highest potential by nurturing leadership, critical thinking, and an appreciation for learning. We advance cultural, social, environmental, and economic well-being for all we serve.

\section{Vision}

\section{HOMETOWN CAMPUS, GLOBAL IMPACT}

The University of South Florida Sarasota-Manatee aspires to be a leader in higher education, delivering accessible academic programs and contributing significant research, while inspiring continuous improvement locally and globally.

\section{Other Contextual Introductory Comments}

USF Sarasota-Manatee was granted separate accreditation from the Southern Association of Colleges and Schools (SACS) in 2011.

\section{BOARD OF GOVERNORS - STATE UNIVERSITY SYSTEM GOAL 1: ACCESS TO AND PRODUCTION OF DEGREES}

\section{Degree Production}

USF Sarasota-Manatee began the process of seeking separate accreditation in 2009. In Fall 2009, USF Tampa mandated that any USF Sarasota-Manatee student in degree programs hosted by USF Tampa or not offered completely by USF SarasotaManatee be removed from USF Sarasota-Manatee Home Campus enrollment numbers. As a result, the number of degrees awarded by USF Sarasota-Manatee dropped significantly in 2009-10; however, in 2010-11 the number of undergraduate degrees awarded was again on par with the 2008-09 levels, a 21\% increase over 2009-10. This recovery is due to the hard work of the faculty and staff of USF SarasotaManatee. For example, Student Services led efforts in the retention and success of current students while Academic Affairs worked to streamline degree programs and to add new programs that aligned with community demand.

USF Sarasota-Manatee selected a peer group in 2010-11.

Benchmarking of data comparisons to this group began in the following Work Plan cycle.

\section{$\underline{\text { Access }}$}

USF Sarasota-Manatee plans to add freshman and sophomore programs in the future to improve access to four-year degrees for the nearly 1,300 high school graduates within Sarasota, Manatee, and De Soto counties who now leave the service area to attend Florida state universities. The addition of the full four-year program is also likely to be attractive to those with financial difficulties, with the need to work full-time, and for 
minorities who often prefer to stay close to their families and communities.

USF Sarasota-Manatee student diversity mirrors that of its local service area; however, the institution has worked diligently to improve access to the university for underrepresented minority groups. For instance, the enrollment of Hispanic students made up 7\% of the reported ethnicities attending USF Sarasota-Manatee in 2006-07 that number increased to $9 \%$ of the enrollment in 2010-11.

\section{BOARD OF GOVERNORS - STATE UNIVERSITY SYSTEM GOAL 2: MEETING STATEWIDE PROFESSIONAL AND WORKFORCE NEEDS}

In alignment with the Board of Governors identified areas of programmatic strategic emphasis, USF Sarasota-Manatee offers degree programs in the critical needs areas of information technology and criminology as well as serving the economic development of its service region by offering degree programs demanded by the regional workforce.

In 2011, USF Sarasota-Manatee received approval to offer the second bachelor's in communication sciences and disorders. This degree will specifically target the statewide shortage of speech-language pathologists. The University also plans to partner with USF Tampa campus to offer the complementary master's degree in this area as well. Additionally in 2011, USF Sarasota-Manatee put forth a proposal to re-instate master's level programs in secondary English education. These programs fall under a Florida Department of Education defined critical teacher shortage area.

\section{BOARD OF GOVERNORS - STATE UNIVERSITY SYSTEM GOAL 3: BUILDING WORLD-CLASS ACADEMIC PROGRAMS AND RESEARCH CAPACITY}

USF Sarasota-Manatee faculty members are committed to conducting relevant research that will contribute to the public good. During the academic year 2010-11, faculty devoted between $5 \%$ and $50 \%$ of their workloads to research efforts and submitted nearly $\$ 600,000$ in grants/proposals. The following are but a few highlights of their efforts:

- USFSM College of Education sponsored the 2010 Children's Literature Symposium - a joint conference with West Central Region F.A.T.E. with the theme of Engaging a New Generation of Readers: The Value of Evolution of Story. The symposium brought presentations from educators, scholars, actors, education consultants, critics, librarians and graduate students to address shifts and developments in literature and explore emerging genres.

- A College of Education professor, as a Co-PI, was awarded a grant from the Social Science Research and Humanities Council of Canada to research children affected by war in northern Uganda. She will be traveling to Uganda in November.

- USF Sarasota-Manatee received a \$131,566 sub-contract from Sarasota County Openly Plans for Excellence (SCOPE) to conduct research for The Aging with Dignity and Independence Initiative, a multi-year project with an end goal of creating innovative and technological approaches to help people 65 years and 
older age with dignity and independence in the community. The research will engage residents through community forums, focus groups and surveys, seeking to learn from the experiences of older adults as well as the broader community. The Aging with Dignity and Independence Initiative is funded by The Patterson Foundation.

\section{BOARD OF GOVERNORS - STATE UNIVERSITY SYSTEM GOAL 4: MEETING COMMUNITY NEEDS AND FULFILLING UNIQUE INSTITUTIONAL \\ RESPONSIBILITIES}

USF Sarasota-Manatee is committed to serving the needs of Sarasota, Manatee, and DeSoto counties. Part of that commitment is the campus' mission to advance the "economic well-being" of our surrounding communities. The following recent efforts evidence this commitment:

- Fall 2010 marked the grand opening of the new USF Sarasota-Manatee @ North Port instructional site. The site, originally housed on the State College of Florida's Venice campus, moved into the newly renovated building to accommodate the ever-steady increase of south Sarasota county student enrollments. The site offers course work towards degrees in criminology, psychology, interdisciplinary social sciences, general business administration, elementary education, and educational leadership.

- USF Sarasota-Manatee received a one-year, $\$ 247,500$ grant from the Health Resources and Services Administration (HRSA) entitled, "A Blueprint for
Health Professions Education at USF SarasotaManatee." Outcomes include a five-year strategic plan for delivery of health care degrees/certificates that meet workforce needs in Sarasota and Manatee Counties.

- Twenty-two local professionals received a professional development certificate in Non-profit Management from USF Sarasota-Manatee this past spring. The program, developed by USFSM and The Community Foundation of Sarasota County, was a ten-week course for potential and current nonprofit managers and leaders to prepare emerging professionals for the unique challenges in today's nonprofit climate.

- USFSM's annual Brunch on the Bay has raised over $\$ 2.7$ million dollars since 1994 to support endowed and First Generation student scholarships.

\section{PROGRESS ON PRIMARY INSTITUTIONAL GOALS AND METRICS AS OUTLINED IN THE UNIVERSITY WORK PLAN}

1. Accreditation: Work toward institutional autonomy within the USF system with separate regional professional accreditations.

In 2011, USFSM was awarded separate regional accreditation from the Southern Association of Colleges and Schools. USFSM is now focused on separate professional accreditations from AACSB and NCATE. 
2. Financial Stability: Grow and diversify the resource base.

USF Sarasota-Manatee lost \$5.96 million in recurring funding from the State of Florida over the last three years. Though the campus sought \$2 million from the legislature in 2010-11 it was not included in the State's spending bill.

3. Assessment: Expand the system of self-study and continuous improvement.

USF Sarasota-Manatee is committed to engaging in selfassessment in both its academic programs and administrative units. The SACS accreditation site visit team cited assessment as a major strength of USFSM. 2010-11 marked the fourth cycle of academic assessment within the newly autonomous USFSM Colleges.

\section{ADDITIONAL INFORMATION ON QUALITY, RESOURCES, EFFICIENCIES, AND EFFECTIVENESS}

In addition to reducing the campus' electrical KWH demand by $21 \%$ since $2006-07$, USFSM Facilities Planning \& Management was also awarded two grants in 2011: 1) Florida Department of Education State Homeland Security Grant Program award of $\$ 50,000$ to install a campus emergency mass notification system and 2) Florida Power \& Light, Energy Efficiency Award Program award of $\$ 150,720$ for Thermal Storage for Central Energy Plant.

\section{ADDITIONAL RESOURCES}

University Strategic Plan http://www.sarasota.usf.edu/ir/Strategic_Plan/StrategicPlan Home.php

USF Sarasota-Manatee 2011 Work Plan http://www.ods.usf.edu/Plans/Strategic/docs/2011-SUS-WorkPlan-USF-Sarasota-Manatee.pdf

\section{USFSM VSA Portrait}

http://www.collegeportraits.org/FL/USFSM

\section{USFSM Common Data Set}

http://www.sarasota.usf.edu/IR/Reports/CDS2010_2011_Marc $\underline{\text { h162011.pdf }}$

\section{College Navigator}

http://nces.ed.gov/collegenavigator $/$ ?q=South+Florida\&s=al $1 \&$ id $=451671$ \#fedloans

\section{USFSM Institutional Peers}

Georgia Southwestern State University Indiana University - Kokomo

Louisiana State University - Shreveport

University of Houston - Victoria

\section{USFSM Aspirational Peers}

Auburn University at Montgomery

University of Houston at Clear Lake

University of North Carolina at Asheville

The University of Texas of the Permian Basin

University Institutional Research Unit http:// www.sarasota.usf.edu/ir/ 
Section 1 - Financial Resources

TABLE 1A. University Education and General Revenues

\begin{tabular}{|c|c|c|c|c|c|}
\hline & $\begin{array}{c}\text { 2007-08 } \\
\text { Actual }\end{array}$ & $\begin{array}{l}\text { 2008-09 } \\
\text { Actual }\end{array}$ & $\begin{array}{l}\text { 2009-10 } \\
\text { Actual }\end{array}$ & $\begin{array}{r}2010-11 \\
\text { Actual }\end{array}$ & $\begin{array}{c}2011-12 \\
\text { Estimates }\end{array}$ \\
\hline $\begin{array}{l}\text { Recurring } \\
\text { State Funds } \\
\text { (GR \& Lottery) }\end{array}$ & $\$ 14,941,901$ & $\$ 13,959,366$ & $\$ 11,996,133$ & $\$ 11,882,443$ & $\$ 11,102,286$ \\
\hline $\begin{array}{l}\text { Non-Recurring } \\
\text { State Funds } \\
\text { (GR \& Lottery) }\end{array}$ & $\$ 150,120$ & $\$ 146,382$ & $\$ 85,400$ & $\$ 93,391$ & $\$ 127,464$ \\
\hline $\begin{array}{l}\text { Tuition } \\
\text { (Resident \& Non-Resident) }\end{array}$ & $\$ 4,184,045$ & $\$ 4,782,090$ & $\$ 5,234,584$ & $\$ 5,766,517$ & $\$ 7,274,243$ \\
\hline Tuition Differential Fee & $\$ 0$ & $\$ 0$ & $\$ 193,210$ & $\$ 498,603$ & $\$ 750,079$ \\
\hline $\begin{array}{l}\text { Other Revenues } \\
\text { (Includes Misc. Fees \& Fines) }\end{array}$ & $\$ 7,722$ & $\$ 8,803$ & $\$ 66,227$ & $\$ 69,897$ & $\$ 96,253$ \\
\hline $\begin{array}{l}\text { Phosphate Research } \\
\text { Trust Fund }\end{array}$ & $\$ 0$ & $\$ 0$ & $\$ 0$ & $\$ 0$ & $\$ 0$ \\
\hline Federal Stimulus Funds & $\$ 0$ & $\$ 0$ & $\$ 1,012,126$ & $\$ 968,456$ & $\$ 0$ \\
\hline TOTAL & $\$ 19,283,788$ & $\$ 18,896,641$ & $\$ 18,587,680$ & $\$ 19,279,307$ & $\$ 19,350,325$ \\
\hline
\end{tabular}

TABLE 1B. University Education and General Expenditures

\begin{tabular}{|c|c|c|c|c|c|}
\hline & $\begin{array}{c}2007-08 \\
\text { Actual }\end{array}$ & $\begin{array}{r}2008-09 \\
\text { Actual }\end{array}$ & $\begin{array}{r}2009-10 \\
\text { Actual }\end{array}$ & $\begin{array}{c}2010-11 \\
\text { Actual }\end{array}$ & $\begin{array}{c}2011-12 \\
\text { Estimates }\end{array}$ \\
\hline Instruction/Research & $\$ 10,457,953$ & $\$ 9,423,525$ & $\$ 9,786,291$ & $\$ 9,995,147$ & $\$ 10,943,768$ \\
\hline $\begin{array}{l}\text { Institutes and Research } \\
\text { Centers }\end{array}$ & $\$ 0$ & $\$ 0$ & $\$ 0$ & $\$ 0$ & $\$ 0$ \\
\hline PO\&M & $\$ 1,268,101$ & $\$ 837,867$ & $\$ 920,903$ & $\$ 1,331,348$ & $\$ 1,185,816$ \\
\hline $\begin{array}{l}\text { Administration and } \\
\text { Support Services }\end{array}$ & $\$ 2,282,003$ & $\$ 2,154,447$ & $\$ 2,528,316$ & $\$ 2,526,918$ & $\$ 4,043,298$ \\
\hline Radio/TV & $\$ 0$ & $\$ 0$ & $\$ 0$ & $\$ 0$ & $\$ 0$ \\
\hline Library/Audio Visual & $\$ 707,931$ & $\$ 545,797$ & $\$ 1,191,766$ & $\$ 363,719$ & $\$ 1,159,358$ \\
\hline Museums and Galleries & $\$ 0$ & $\$ 0$ & $\$ 0$ & $\$ 0$ & $\$ 0$ \\
\hline Agricultural Extension & $\$ 0$ & $\$ 0$ & $\$ 0$ & $\$ 0$ & $\$ 0$ \\
\hline Student Services & $\$ 2,493,848$ & $\$ 1,829,878$ & $\$ 1,829,151$ & $\$ 1,859,587$ & $\$ 1,995,835$ \\
\hline Intercollegiate Athletics & $\$ 0$ & $\$ 0$ & $\$ 0$ & $\$ 0$ & $\$ 0$ \\
\hline $\begin{array}{l}\text { Academic Infrastructure } \\
\text { Support Organization }\end{array}$ & $\$ 0$ & $\$ 0$ & $\$ 0$ & $\$ 0$ & $\$ 0$ \\
\hline TOTAL & $\$ 17,209,836$ & $\$ 14,791,514$ & $\$ 16,256,427$ & $\$ 16,076,719$ & $\$ 19,328,075$ \\
\hline
\end{tabular}

The table reports the actual and estimated amount of expenditures from revenues appropriated by the Legislature for each fiscal year. The expenditures are classified by Program Component (i.e.

Instruction/Research, PO\&M, Administration, etc.) for activities directly related to instruction, research

and public service. The table does not include expenditures classified as non-operating expenditures

(i.e., to service asset-related debts), and therefore excludes a small portion of the amount appropriated

each year by the Legislature. Also, the table does not include expenditures from funds carried forward from previous years.

USF Sarasota-Manatee 2011 Annual Report
Section 1 - Financial Resources (continued)

\begin{tabular}{|c|c|c|c|c|c|}
\hline & $\begin{array}{c}2007-08 \\
\text { Actual }\end{array}$ & $\begin{array}{c}2008-09 \\
\text { Actual }\end{array}$ & $\begin{array}{c}2009-10 \\
\text { Actual }\end{array}$ & $\begin{array}{c}2010-11 \\
\text { Actual }\end{array}$ & $\begin{array}{c}2011-12 \\
\text { Estimates }\end{array}$ \\
\hline \multicolumn{6}{|c|}{ Appropriated Funding per FTE } \\
\hline General Revenue per FTE & $\$ 10,227$ & $\$ 8,813$ & $\$ 7,130$ & $\$ 6,840$ & $\$ 6,136$ \\
\hline Lottery Funds per FTE & $\$ 438$ & $\$ 693$ & $\$ 546$ & $\$ 659$ & $\$ 741$ \\
\hline Tuition \& Fees per FTE & $\$ 2,962$ & $\$ 3,228$ & $\$ 3,490$ & $\$ 4,560$ & $\$ 4,973$ \\
\hline Other Trust Funds per FTE & $\$ 0$ & $\$ 0$ & $\$ 643$ & $\$ 606$ & $\$ 0$ \\
\hline Total per FTE & $\$ 13,627$ & $\$ 12,734$ & $\$ 11,809$ & $\$ 12,665$ & $\$ 11,850$ \\
\hline \multicolumn{6}{|l|}{ Actual Funding per FTE } \\
\hline Tuition \& Fees per FTE & $\$ 2,962$ & $\$ 3,228$ & $\$ 3,490$ & $\$ 3,967$ & $\$ 4,973$ \\
\hline Total per FTE & $\$ 13,627$ & $\$ 12,734$ & $\$ 11,809$ & $\$ 12,072$ & $\$ 11,850$ \\
\hline \multicolumn{6}{|c|}{$\begin{array}{l}\text { Notes: (1) FTE is based on actual FTE, not funded FTE; (2) does not include Health-Science Center funds } \\
\text { or FTE; (3) FTE for these metrics uses the standard IPEDS definition of FTE, equal to } 30 \text { credit hours for } \\
\text { undergraduates and } 24 \text { for graduates; and (4) actual funding per student is based on actual tuition and } \\
\text { E\&G fees (does not include local fees) collected. }\end{array}$} \\
\hline
\end{tabular}

\begin{tabular}{|c|c|c|c|c|c|}
\hline & $\begin{array}{r}2007-08 \\
\text { Actual }\end{array}$ & $\begin{array}{r}2008-09 \\
\text { Actual }\end{array}$ & $\begin{array}{r}2009-10 \\
\text { Actual } \\
\end{array}$ & $\begin{array}{r}2010-11 \\
\text { Actual } \\
\end{array}$ & $\begin{array}{c}2011-12 \\
\text { Estimates }\end{array}$ \\
\hline \multicolumn{6}{|c|}{ Auxiliary Enterprises } \\
\hline $\begin{array}{l}\text { Revenues } \\
\text { Expenditures }\end{array}$ & \multicolumn{5}{|c|}{$\begin{array}{l}\text { Contracts \& Grants data is a USF system-wide function } \\
\text { and consolidated system data is available at this time. }\end{array}$} \\
\hline \multicolumn{6}{|c|}{ Contracts \& Grants } \\
\hline $\begin{array}{l}\text { Revenues } \\
\text { Expenditures } \\
\end{array}$ & \multicolumn{5}{|c|}{$\begin{array}{l}\text { Contracts \& Grants data is a USF system-wide function } \\
\text { and consolidated system data is available at this time. }\end{array}$} \\
\hline \multicolumn{6}{|l|}{ Local Funds } \\
\hline $\begin{array}{l}\text { Revenues } \\
\text { Expenditures }\end{array}$ & \multicolumn{5}{|c|}{$\begin{array}{l}\text { Contracts \& Grants data is a USF system-wide function } \\
\text { and consolidated system data is available at this time. }\end{array}$} \\
\hline
\end{tabular}

\begin{tabular}{|c|c|c|c|c|c|}
\hline & $\begin{array}{c}2007-08 \\
\text { Actual } \\
\end{array}$ & $\begin{array}{c}\text { 2008-09 } \\
\text { Actual } \\
\end{array}$ & $\begin{array}{c}\text { 2009-10 } \\
\text { Actual } \\
\end{array}$ & $\begin{array}{c}\text { 2010-11 } \\
\text { Actual }\end{array}$ & $\begin{array}{c}2011-12 \\
\text { Estimates }\end{array}$ \\
\hline $\begin{array}{c}\text { Total } \\
\text { Revenues }\end{array}$ & $\$ 19,283,788$ & $\$ 18,896,641$ & $\$ 18,587,680$ & $\$ 19,279,307$ & $\$ 19,350,325$ \\
\hline $\begin{array}{c}\text { Total } \\
\text { Expenditures }\end{array}$ & $\$ 17,209,836$ & $\$ 14,791,514$ & $\$ 16,256,427$ & $\$ 16,076,719$ & $\$ 19,328,075$ \\
\hline
\end{tabular}




\section{Section 1 - Financial Resources (continued)}

TABLE 1F. Voluntary Support of Higher Education

Endowment Market

Value (Thousand \$)

Annual Gifts

Received (\$)

Percentage of

Graduates Who are

Alumni Donors

\begin{tabular}{|l|c|c|}
\hline TABLE 1G. University Federal Stimulus Dollars (ARRA) & $\begin{array}{c}\mathbf{2 0 1 0 - 1 1} \\
\text { Actual }\end{array}$ \\
\hline Jobs Saved/Created & $\begin{array}{r}\mathbf{2 0 0 9 - 1 0} \\
\text { Actual }\end{array}$ & $\$ 968,456$ \\
\hline Scholarships & $\$ 1,012,126$ & $\$ 0$ \\
\hline Library Resources & $\$ 0$ & $\$ 0$ \\
\hline Building Repairs/Alterations & $\$ 0$ & $\$ 0$ \\
\hline Motor Vehicles & $\$ 0$ & $\$ 0$ \\
\hline Printing & $\$ 0$ & $\$ 0$ \\
\hline Furniture \& Equipment & $\$ 0$ & $\$ 0$ \\
\hline Information Technology Equipment & $\$ 0$ & $\$ 0$ \\
\hline Financial Aid to Medical Students & $\$ 0$ & $\$ 0$ \\
\hline Other & $\$ 0$ & $\$ 0$ \\
\hline TOTAL & $\$ 0$ & $\$ \mathbf{9 6 8 , 4 5 6}$ \\
\hline
\end{tabular}

\section{Section 2 - Personnel}

\section{TABLE 2A. Personnel Headcount}

\begin{tabular}{|c|c|c|c|c|c|c|c|c|c|c|}
\hline & \multicolumn{2}{|c|}{ Fall 2006} & \multicolumn{2}{|c|}{ Fall 2007} & \multicolumn{2}{|c|}{ Fall 2008} & \multicolumn{2}{|c|}{ Fall 2009} & \multicolumn{2}{|c|}{ Fall 2010} \\
\hline & $\begin{array}{l}\text { Full- } \\
\text { Time }\end{array}$ & $\begin{array}{l}\text { Part- } \\
\text { Time }\end{array}$ & $\begin{array}{l}\text { Full- } \\
\text { Time }\end{array}$ & $\begin{array}{l}\text { Part- } \\
\text { Time }\end{array}$ & $\begin{array}{l}\text { Full- } \\
\text { Time }\end{array}$ & $\begin{array}{l}\text { Part- } \\
\text { Time }\end{array}$ & $\begin{array}{l}\text { Full- } \\
\text { Time }\end{array}$ & $\begin{array}{l}\text { Part- } \\
\text { Time }\end{array}$ & $\begin{array}{l}\text { Full- } \\
\text { Time }\end{array}$ & $\begin{array}{l}\text { Part- } \\
\text { Time }\end{array}$ \\
\hline $\begin{array}{l}\text { Total Tenure/ } \\
\text { Tenure-track } \\
\text { Faculty }\end{array}$ & 26 & 2 & 25 & 1 & 28 & 1 & 31 & 1 & 32 & 1 \\
\hline $\begin{array}{c}\text { Total Non- } \\
\text { Tenure Track } \\
\text { Faculty }\end{array}$ & 16 & 10 & 14 & 11 & 22 & 8 & 21 & 4 & 17 & 86 \\
\hline $\begin{array}{c}\text { Instructors } \\
\text { Without Faculty } \\
\text { Status }\end{array}$ & 0 & 0 & 0 & 0 & 0 & 0 & 0 & 0 & 0 & 0 \\
\hline $\begin{array}{c}\text { Total Graduate } \\
\text { Assistants/ } \\
\text { Associates }\end{array}$ & 0 & 8 & 0 & 8 & 0 & 5 & 0 & 2 & 0 & 4 \\
\hline $\begin{array}{c}\text { Total Executive/ } \\
\text { Administrative/ } \\
\text { Managerial }\end{array}$ & 15 & 0 & 25 & 0 & 23 & 0 & 27 & 0 & 26 & 0 \\
\hline $\begin{array}{c}\text { Total Other } \\
\text { Professional }\end{array}$ & 40 & 2 & 33 & 2 & 38 & 2 & 34 & 1 & 35 & 0 \\
\hline $\begin{array}{c}\text { Total Non- } \\
\text { Professional }\end{array}$ & 33 & 0 & 45 & 0 & 38 & 0 & 38 & 0 & 40 & 0 \\
\hline TOTAL & \multicolumn{2}{|c|}{152} & \multicolumn{2}{|c|}{164} & \multicolumn{2}{|c|}{165} & \multicolumn{2}{|c|}{159} & \multicolumn{2}{|c|}{241} \\
\hline
\end{tabular}


Section 3 - Enrollment

\section{TABLE 3A. University Full-Time Enrollment (FTE)}

2009-10

$$
\text { 2010-11 }
$$

2011-12

\begin{tabular}{|c|c|c|c|c|c|c|}
\hline 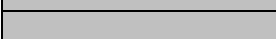 & Funded & Actual & Funded & Actual & Funded & Estimated \\
\hline
\end{tabular}

\begin{tabular}{|c|c|c|c|c|c|c|}
\hline Lower & 0 & 32 & 0 & 64 & 0 & 115 \\
\hline Upper & 798 & 968 & 798 & 984 & 798 & 972 \\
\hline Grad I & 182 & 160 & 182 & 123 & 182 & 111 \\
\hline Grad II & 0 & 1 & 0 & 2 & 0 & 1 \\
\hline Total & $\mathbf{9 8 0}$ & $\mathbf{1 , 1 6 2}$ & $\mathbf{9 8 0}$ & $\mathbf{1 , 1 7 2}$ & $\mathbf{9 8 0}$ & $\mathbf{1 , 1 9 9}$ \\
\hline
\end{tabular}

\section{$\begin{array}{cc}\text { Total } & 980 \\ \text { NON-FLORIDA RESIDENTS }\end{array}$}

\begin{tabular}{|c|c|c|c|c|c|c|}
\hline Lower & 0 & & 2 & & 3 \\
\hline Upper & 16 & & 21 & & 20 \\
\hline Grad I & 3 & & 2 & & 2 \\
\hline Grad II & & 0 & & 0 & & 0 \\
\hline Total & $\mathbf{1 9}$ & & $\mathbf{2 5}$ & & $\mathbf{2 5}$ \\
\hline
\end{tabular}

\section{TOTAL FTE}

\begin{tabular}{|l|c|c|c|c|c|c|}
\hline TOTAL FTE & 0 & 33 & 0 & 66 & 0 & 119 \\
\hline Lower & 798 & 984 & 798 & 1,005 & 798 & 991 \\
\hline Upper & 182 & 162 & 182 & 125 & 182 & 113 \\
\hline Grad I & 0 & 1 & 0 & 2 & 0 & 1 \\
\hline $\begin{array}{l}\text { Grad II } \\
\text { (FL Definition) }\end{array}$ & $\mathbf{9 8 0}$ & $\mathbf{1 , 1 8 1}$ & $\mathbf{9 8 0}$ & $\mathbf{1 , 1 9 8}$ & $\mathbf{9 8 0}$ & $\mathbf{1 , 2 2 5}$ \\
\hline $\begin{array}{l}\text { Total FTE } \\
\text { (US Definition) }\end{array}$ & $\mathbf{1 , 3 0 7}$ & $\mathbf{1 , 5 7 4}$ & $\mathbf{1 , 3 0 7}$ & $\mathbf{1 , 5 9 7}$ & $\mathbf{1 , 3 0 7}$ & $\mathbf{1 , 6 3 3}$ \\
\hline
\end{tabular}

Notes: Florida definitions of FTE (Undergraduate FTE $=40$ and Graduate FTE $=32$ credit hours per FTE) are used for all items except the row named Total FTE (US Definition), which is based on an

Undergraduate FTE $=30$ and Graduate FTE $=24$ credit hours.

\section{Section 3 - Enrollment (continued)}

TABLE 3B. Enrollment by Location

\begin{tabular}{|c|c|c|c|}
\hline & $\begin{array}{l}\text { 2009-10 } \\
\text { Actual }\end{array}$ & $\begin{array}{r}2010-11 \\
\text { Actual }\end{array}$ & $\begin{array}{c}2011-12 \\
\text { Estimated }\end{array}$ \\
\hline \multicolumn{4}{|l|}{ MAIN CAMPUS } \\
\hline Lower & 16 & 41 & 77 \\
\hline Upper & 577 & 535 & 438 \\
\hline Grad I & 126 & 100 & 89 \\
\hline Grad II & 0 & 0 & 0 \\
\hline TOTAL & 719 & 677 & 604 \\
\hline
\end{tabular}

\section{VIRTUAL/DISTANCE LEARNING}

For the sum of current or planned State-fundable FTE enrollments not served at a physical location.

\begin{tabular}{|c|c|c|c|}
\hline Lower & 17 & 25 & 42 \\
\hline Upper & 407 & 469 & 553 \\
\hline Grad I & 37 & 25 & 25 \\
\hline Grad II & 1 & 1 & 1 \\
\hline TOTAL & $\mathbf{4 6 1}$ & $\mathbf{5 2 1}$ & $\mathbf{6 2 1}$ \\
\hline
\end{tabular}

Note: The FTE reported in this table are more than the total in table 3A (which does not include HSC FTE). Estimated figures-actual enrollment to be finalized in Spring 2012 
Section 4 - Undergraduate Education

TABLE 4A. Baccalaureate Degree Program Changes in AY 2010-11

\begin{tabular}{|l|l|l|l|l|}
\hline New Programs & N/A & & & \\
\hline Terminated Programs & N/A & & & \\
\hline \multicolumn{2}{|l|}{} & & & \\
\hline Inactive Programs & N/A & & & \\
\hline
\end{tabular}

New Programs Considered By University But Not Approved

$\mathrm{N} / \mathrm{A}$

Note: This table does not include new majors or concentrations added under an existing degree program CIP Code. This table reports the program changes between May 5, 2010 and May 4, 2011. New

Programs are proposed new degree programs that have been completely through the approval process

at the university and, if appropriate, the Board of Governors. Terminated Programs are degree programs for which the entire CIP Code has been terminated and removed from the university's

inventory of degree programs. Inactive Programs are degree programs for which enrollments have been temporarily suspended for the entire CIP Code, but the program CIP Code has not been terminated.

\section{TABLE 4B. First-Year Persistence Rates}

\begin{tabular}{|c|c|c|c|c|c|}
\hline $\begin{array}{c}\text { Term of Entry } \\
\text { Cohort Size } \\
\text { Full-time FTIC }\end{array}$ & Fall 2005 & Fall 2006 & Fall 2007 & Fall 2008 & $\begin{array}{c}\text { Fall 2009 } \\
\text { Preliminary }\end{array}$ \\
\hline From Same University \\
\hline \% Still Enrolled & N/A; No FTICs \\
\hline
\end{tabular}

TABLE 4C. Federal Definition - Undergraduate Progression and Graduation Rates for Full-Time First-Time-in-College (FTIC) Students

\begin{tabular}{|c|c|c|c|c|c|}
\hline Term of Entry & Fall 2001 & Fall 2002 & Fall 2003 & Fall 2004 & $\begin{array}{c}\text { Fall } 2005 \\
\text { Preliminary }\end{array}$ \\
\hline Cohort Size & & &
\end{tabular}

Cohort Size

N/A; No FTICs

\section{6 - Year Rates}

\section{From Same University}

$\%$ Graduated

$\%$ Still Enrolled

N/A; No FTICs

$\%$ Success Rate

Notes: (1) Cohorts are based on undergraduate students who enter the institution in the Fall term (or

Summer term and continue into the Fall term); (2) Success Rate measures the percentage of an initial

cohort of students who have either graduated or are still enrolled; (3) since degrees can be awarded after the last semester of coursework, the most recent year of data in this table provides preliminary

graduation rate data that may change with the addition of "late degrees". Late degrees reported in

conjunction with the IPEDS Graduation Rate Survey due in mid-April will be reflected in the following

year.

USF Sarasota-Manatee 2011 Annual Report
Section 4 - Undergraduate Education (continued)

TABLE 4D. SUS Definition - Undergraduate Progression and Graduation Rates

for First-Time-in-College (FTIC) Students

\begin{tabular}{|c|c|c|c|c|c|}
\hline Term of Entry & Fall 2001 & Fall 2002 & Fall 2003 & Fall 2004 & $\begin{array}{l}\text { Fall } 200 \\
\text { Preliminar }\end{array}$ \\
\hline $\begin{array}{c}\text { Cohort Size } \\
\text { Full- E Part-time }\end{array}$ & \multicolumn{5}{|c|}{ N/A; No FTICs } \\
\hline
\end{tabular}

\section{4 - Year Rates}

From Same University

\begin{tabular}{l|l} 
\% Graduated & \\
\cline { 1 - 1 } \% Still Enrolled & N/A; No FTICs
\end{tabular}

From Other SUS University

\begin{tabular}{l|l}
$\%$ Graduated & N/A; No FTICs
\end{tabular}

$\%$ Still Enrolled

N/A; No FTICs

From State University System

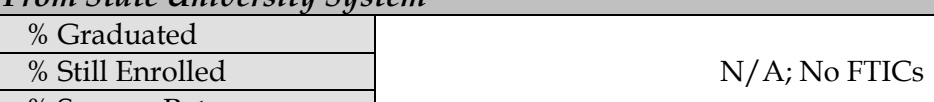

\section{6 - Year Rates}

From Same University

\begin{tabular}{l|l}
$\%$ Graduated & N/A; No FTICs
\end{tabular}

$\%$ Still Enrolled

From Other SUS University

$\%$ Graduated

$\%$ Still Enrolled

N/A; No FTICs

From State University System

$\%$ Graduated

$\%$ Still Enrolled

$\%$ Success Rate

Notes: (1) Cohorts are based on undergraduate students who enter the institution in the Fall term (or

Summer term and continue into the Fall term); (2) Success Rate measures the percentage of an initial

cohort of students who have either graduated or are still enrolled; (3) since degrees can be awarded after

the last semester of coursework, the most recent year of data in this table provides preliminary

graduation rate data that may change with the addition of "late degrees". Late degrees reported in

conjunction with the IPEDS Graduation Rate Survey due in mid-April will be reflected in the following year. 


\section{Section 4 - Undergraduate Education (continued)}

TABLE 4E. SUS Definition - Undergraduate Progression and Graduation Rates

for AA Transfer Students

\begin{tabular}{|c|c|c|c|c|c|}
\hline Term of Entry & Fall 2003 & Fall 2004 & Fall 2005 & Fall 2006 & $\begin{array}{l}\text { Fall } 2007 \\
\text { Preliminary }\end{array}$ \\
\hline $\begin{array}{l}\text { Cohort Size } \\
\text { Full- \& Part-time }\end{array}$ & \multicolumn{5}{|c|}{$\begin{array}{l}\text { Parsing of retention/graduation data at the campus-level cannot be } \\
\text { completed at this time. }\end{array}$} \\
\hline
\end{tabular}

\section{2 - Year Rates}

From Same University

$\%$ Graduated

$\%$ Still Enrolled

Parsing of retention/graduation data at the campus-level cannot be completed at this time.

\section{From Other SUS University}

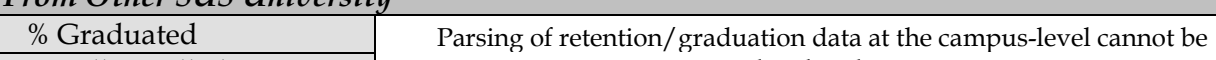

$\%$ Still Enrolled

From State University System

$\%$ Graduated

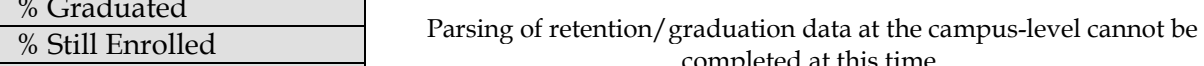

$\%$ Success Rate

4 - Year Rates

From Same University

$\%$ Graduated

$\%$ Still Enrolled

Parsing of retention/graduation data at the campus-level cannot be

From Other SUS University

$\%$ Graduated

$\%$ Still Enrolled

completed at this time.

From State University System

$\%$ Graduated

$\%$ Still Enrolled

$\%$ Success Rate

Notes: (1) Cohorts are based on undergraduate students who enter the institution in the Fall term (or Summer term and continue into the Fall term); (2) Success Rate measures the percentage of an initial cohort of students who have either graduated or are still enrolled; (3) since degrees can be awarded after the last semester of coursework, the most recent year of data in this table provides preliminary graduation rate data that may change with the addition of "late degrees". Late degrees reported in conjunction with the IPEDS Graduation Rate Survey due in mid-April will be reflected in the following year.

Parsing of retention/graduation data at the campus-level cannot be completed at this time.

USF Sarasota-Manatee 2011 Annual Report

\section{Section 4 - Undergraduate Education (continued)}

TABLE 4F. SUS Definition - Undergraduate Progression and Graduation Rates

for Other Transfer Students

\begin{tabular}{c|c|c|c|c|c|}
$\begin{array}{c}\text { Term of Entry } \\
\text { Cohort Size }\end{array}$ & Fall 2002 & Fall 2003 & Fall 2004 & Fall 2005 & $\begin{array}{c}\text { Fall } 2006 \\
\text { Preliminary }\end{array}$ \\
\hline \multicolumn{3}{|c|}{ Parsing of retention/graduation data at the campus-level cannot be }
\end{tabular}

Full- E Part-time completed at this time.

\section{5 - Year Rates}

$\%$ Graduated

$\%$ Still Enrolled

Parsing of retention/graduation data at the campus-level cannot be

From Other SUS University

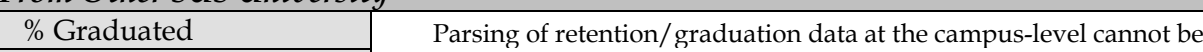

$\%$ Still Enrolled

Parsing of retention/graduation data at the
completed at this time.

From State University System

$\%$ Graduated

\% Still Enrolled Parsing of retention/graduation data at the campus-level cannot be

$\%$ Success Rate

Notes: (1) Cohorts are based on undergraduate students who enter the institution in the Fall term (or Summer term and continue into the Fall term); (2) Success Rate measures the percentage of an initial

cohort of students who have either graduated or are still enrolled; (3) since degrees can be awarded after

the last semester of coursework, the most recent year of data in this table provides preliminary

graduation rate data that may change with the addition of "late degrees". Late degrees reported in

conjunction with the IPEDS Graduation Rate Survey due in mid-April will be reflected in the following

year.

\section{TABLE 4G. Baccalaureate Degrees Awarded}

\begin{tabular}{|c|c|c|c|c|c|}
\hline & $\mathbf{2 0 0 6 - 0 7}$ & $\mathbf{2 0 0 7 - 0 8}$ & $\mathbf{2 0 0 8 - 0 9}$ & $\mathbf{2 0 0 9 - 1 0}$ & $\mathbf{2 0 1 0 - 1 1}$ \\
\hline TOTAL & 380 & 427 & 450 & 357 & 453 \\
\hline
\end{tabular}

TABLE 4H. Baccalaureate Degrees Awarded in Areas of Strategic Emphasis

\begin{tabular}{|c|c|c|c|c|c|}
\hline & $\mathbf{2 0 0 6 - 0 7}$ & $\mathbf{2 0 0 7 - 0 8}$ & $\mathbf{2 0 0 8 - 0 9}$ & $\mathbf{2 0 0 9 - 1 0}$ & $\mathbf{2 0 1 0 - 1 1}$ \\
\hline Education & 2 & 10 & 11 & 0 & 0 \\
\hline Health Professions & 6 & 18 & 16 & 4 & 0 \\
\hline $\begin{array}{c}\text { Science, Technology, } \\
\text { Engineering, and Math }\end{array}$ & 12 & 18 & 23 & 10 & 14 \\
\hline $\begin{array}{c}\text { Security and Emergency } \\
\text { Services }\end{array}$ & 27 & 22 & 28 & 37 & 34 \\
\hline Globalization & 8 & 0 & 0 & 0 & 0 \\
\hline SUBTOTAL & $\mathbf{5 5}$ & $\mathbf{6 8}$ & $\mathbf{7 8}$ & $\mathbf{5 1}$ & $\mathbf{4 8}$ \\
\hline
\end{tabular}


Section 4 - Undergraduate Education (continued)

TABLE 4I. Baccalaureate Degrees Awarded to Underrepresented Groups \begin{tabular}{|l|l|l|l|l|}
\hline $2006-07$ & $2007-08$ & $2008-09$ & $2009-10$ \\
\hline
\end{tabular}

2010-11

Non-Hispanic Black Number of

Baccalaureate Degrees

Percentage of All

Baccalaureate Degrees

32

Hispanic

\begin{tabular}{|c|c|c|c|c|c|}
\hline $\begin{array}{c}\text { Hispanic } \\
\text { Number of } \\
\text { Baccalaureate Degrees }\end{array}$ & 18 & 37 & $\begin{array}{c}27 \\
\text { Increase* }\end{array}$ & 26 & 35 \\
\hline $\begin{array}{c}\text { Percentage of All } \\
\text { Baccalaureate Degrees }\end{array}$ & $5 \%$ & $9 \%$ & $\begin{array}{c}6 \% \\
\text { Increase* }\end{array}$ & $8 \%$ & $8 \%$ \\
\hline Pell-Grant Recipients & 138 & 153 & $\begin{array}{c}158 \\
\text { Increase* }\end{array}$ & 161 & 193 \\
\hline $\begin{array}{c}\text { Number of } \\
\text { Baccalaureate Degrees }\end{array}$ & $38 \%$ & $38 \%$ & $\begin{array}{c}36 \% \\
\text { Increase* }\end{array}$ & $46 \%$ & $43 \%$ \\
\hline $\begin{array}{c}\text { Percentage of All } \\
\text { Baccalaureate Degrees }\end{array}$ & n &
\end{tabular}

Note: Pell-Grant recipients are defined as those students who have received a Pell grant from any SUS Institution within six years of graduation. This does not include degrees awarded to students whose race/ethnicity code is missing (or not reported) or for students who are non-resident aliens.

Note*: Directional goals for the 2012-13 year were established in the 2010 University Work Plan.

\section{TABLE 4J. Baccalaureate Completion Without Excess Credit Hours}

\section{$\%$ of Total Baccalaureate} 2006-07

2007-08

\section{8-09}

2009-10

2010-1
Degrees Awarded

Within $110 \%$ of Hours

Required for Degree

$62 \%$

$89 \%$

$66 \%$

$63 \%$

\section{TABLE 4K. Undergraduate Course Offerings}

\begin{tabular}{|c|c|c|c|c|c|}
\hline & Fall 2006 & Fall 2007 & Fall 2008 & Fall 2009 & Fall 2010 \\
\hline $\begin{array}{c}\text { Number of } \\
\text { Course Sections }\end{array}$ & 179 & 193 & 143 & 135 & 136 \\
\hline \begin{tabular}{l} 
Percentage of Undergraduate Course Sections by Class Size \\
\hline Fewer than 30 Students
\end{tabular}$\quad 86 \%$ & $89 \%$ & $82 \%$ & $87 \%$ & $85 \%$ \\
\hline 30 to 49 Students & $13 \%$ & $11 \%$ & $15 \%$ & $11 \%$ & $12 \%$ \\
\hline 50 to 99 Students & $0 \%$ & $0 \%$ & $3 \%$ & $2 \%$ & $3 \%$ \\
\hline 100 or More Students & $1 \%$ & $1 \%$ & $1 \%$ & $1 \%$ & $0 \%$ \\
\hline
\end{tabular}

Section 4 - Undergraduate Education (continued)

\section{TABLE 4L. Faculty Teaching Undergraduates}

2006-07 2007-08

Percentage of Credit Hours Taught by:

\begin{tabular}{|l|c|c|c|c|c|}
\hline Faculty & $56 \%$ & $61 \%$ & $58 \%$ & $60 \%$ & $64 \%$ * \\
\hline
\end{tabular}

Adjunct Faculty

Graduate Students

Other Instructors

$41 \%$

\begin{tabular}{l|c|}
\hline $41 \%$ & $38 \%$ \\
\hline $2 \%$ & $1 \%$
\end{tabular}

$0 \%$

$0 \%$

\begin{tabular}{|l|l}
39 \\
\hline
\end{tabular}

Note: The definition of faculty varies for Tables $4 \mathrm{~L}, 4 \mathrm{M}$ and $4 \mathrm{~N}$. For Faculty Teaching Undergra the definition of faculty is based on pay plans 01,02 , and 22 .

*Data coding and reclassification of faculty in the institutions' source system reflects the $2010-11$ figure of $\mathbf{7 3 \% \text { ; }}$ future reporting from the state file will be in line with internal classification modifications.

\section{TABLE 4M. Undergraduate Instructional Faculty Compensation}

\begin{tabular}{|c|c|c|c|c|c|}
\hline & $\mathbf{2 0 0 6 - 0 7}$ & $\mathbf{2 0 0 7 - 0 8}$ & $\mathbf{2 0 0 8 - 0 9}$ & $\mathbf{2 0 0 9 - 1 0}$ & $\mathbf{2 0 1 0 - 1 1}$ \\
\hline $\begin{array}{l}\text { Average Salary and } \\
\begin{array}{l}\text { Benefits for Faculty } \\
\text { Who Teach at Least One } \\
\text { Undergraduate Course }\end{array}\end{array}$ & $\$ 81,592$ & $\$ 87,377$ & $\$ 86,817$ & $\$ 87,857$ & $\$ 84,390$ \\
\hline $\begin{array}{l}\text { Note: The definition of faculty varies for Tables 4L, 4M and 4N. For Undergraduate Instructional Faculty } \\
\text { Compensation, the definition of faculty is based on pay plan 22. }\end{array}$ \\
\hline
\end{tabular}

\section{TABLE 4N. Student/Faculty Ratio}

\begin{tabular}{|c|c|c|c|c|c|}
\hline . & Fall 2006 & Fall 2007 & Fall 2008 & Fall 2009 & Fall 2010 \\
\hline Student-to-Faculty Ratio & 21 & 26 & 23 & 19 & 15 \\
\hline
\end{tabular}

TABLE 4O. Professional Licensure/Certification Exams for Undergraduate Programs \begin{tabular}{l|l|l|l|}
$2006-07$ & $2007-08$ & $2008-09$ & $2009-10$ \\
\hline
\end{tabular}

Nursing: National Council Licensure Examination for Registered Nurses

Examinees

Pass Rate

N/A

National Benchmark 


\section{Section 4 - Undergraduate Education (continued)}

\section{TABLE 4P. Tuition Differential Fee}

\begin{tabular}{|c|c|c|c|}
\hline $\begin{array}{c}\text { Total Revenues Generated } \\
\text { By the Tuition Differential }\end{array}$ & $\$ 193,210$ & $\$ 498,603$ & $\$ 750,079$ \\
\hline $\begin{array}{c}\text { Unduplicated Count of Students } \\
\text { Receiving a Financial Aid Award } \\
\text { Funded by Tuition Differential Revenues }\end{array}$ & 74 & 188 & \\
\hline $\begin{array}{c}\text { Projected } \\
\text { Average Amount of Awards } \\
\text { Funded by Tuition Differential Revenues } \\
\text { (per student receiving an award) }\end{array}$ & $\$ 842$ & $\$ 796$ & 380 \\
\hline $\begin{array}{c}\text { Number of Students Eligible for a } \\
\text { Florida Student Assistance Grant (FSAG) }\end{array}$ & 336 & 0 & \\
\hline $\begin{array}{c}\text { Number of FSAG-Eligible Students } \\
\text { Receiving a Waiver } \\
\text { of the Tuition Differential }\end{array}$ & 0 & 0 & \\
\hline $\begin{array}{c}\text { Value of Tuition Differential Waivers } \\
\text { Provided to FSAG-Eligible Students }\end{array}$ & 0 & & \\
\hline
\end{tabular}

\section{Section 5 - Graduate Education}

\section{TABLE 5A. Graduate Degree Program Changes in AY 2010-11}

\begin{tabular}{|l|c|c|c|c|c|c|}
\hline \multicolumn{1}{|c|}{ Title of Program } & $\begin{array}{c}\text { Six-digit } \\
\text { CIP } \\
\text { Code }\end{array}$ & $\begin{array}{c}\text { Degree } \\
\text { Level }\end{array}$ & $\begin{array}{c}\text { Date of } \\
\text { UBOT } \\
\text { Action }\end{array}$ & $\begin{array}{c}\text { Starting } \\
\text { or Ending } \\
\text { Term }\end{array}$ & $\begin{array}{c}\text { Date of } \\
\text { Board of } \\
\text { Governors } \\
\text { Action }\end{array}$ & Comments \\
\hline New Programs & \multicolumn{2}{|c|}{} \\
\hline
\end{tabular}

\begin{tabular}{|c|c|c|c|c|c|c}
\hline & & & & USF
\end{tabular}

\begin{tabular}{|l|l|l|l|l|l|l}
\hline Administration/Manageme & 52.0901 & Masters & $10 / 7 / 2010$ & Fall 2011 & & Sarasota- \\
\hline
\end{tabular}

nt, General

Terminated Programs

$\mathrm{N} / \mathrm{A}$

\section{Inactive Programs}

N/A

\section{New Programs Considered By University But Not Approved}

\section{$\mathrm{N} / \mathrm{A}$}

Note: This table does not include new majors or concentrations added under an existing degree program CIP Code. This table reports the program changes between May 5, 2010 and May 4, 2011. New

Programs are proposed new degree programs that have been completely through the approval process at the university and, if appropriate, the Board of Governors. Terminated Programs are degree programs for which the entire CIP Code has been terminated and removed from the university's inventory of degree programs. Inactive Programs are degree programs for which enrollments have been temporarily suspended for the entire CIP Code, but the program CIP Code has not been terminated.

\section{TABLE 5B. Graduate Degrees Awarded}

\begin{tabular}{|l|c|c|c|c|c|}
\hline \multicolumn{1}{|c|}{ TOTAL } & $\mathbf{2 0 0 6 - 0 7}$ & $\mathbf{2 0 0 7 - 0 8}$ & $\mathbf{2 0 0 8 - 0 9}$ & $\mathbf{2 0 0 9 - 1 0}$ & $\mathbf{2 0 1 0 - 1 1}$ \\
\hline Masters and Specialist & $\mathbf{8 6}$ & $\mathbf{1 0 9}$ & $\mathbf{1 2 5}$ & $\mathbf{5 9}$ & $\mathbf{6 8}$ \\
\hline Research Doctoral & 86 & 109 & 125 & 59 & 68 \\
\hline Professional Doctoral & 0 & 0 & 0 & 0 & 0 \\
\hline a) Medicine & 0 & 0 & 0 & 0 & 0 \\
\hline b) Law & 0 & 0 & 0 & 0 & 0 \\
\hline c) Pharmacy & 0 & 0 & 0 & 0 & 0 \\
\hline $\begin{array}{l}\text { Note: The total number of Professional Doctoral degrees includes other programs that are not } \\
\text { specifically identified in lines a, b, and c. }\end{array}$
\end{tabular}




\section{Section 5 - Graduate Education (continued)}

TABLE 5C. Graduate Degrees Awarded in Areas of Strategic Emphasis

\begin{tabular}{|c|c|c|c|c|c|}
\hline & $\mathbf{2 0 0 6 - 0 7}$ & $\mathbf{2 0 0 7 - 0 8}$ & $\mathbf{2 0 0 8 - 0 9}$ & $\mathbf{2 0 0 9 - 1 0}$ & $\mathbf{2 0 1 0 - 1 1}$ \\
\hline Education & $\mathbf{2 6}$ & 19 & 13 & 12 & 19 \\
\hline Health Professions & 3 & 9 & 7 & 4 & 0 \\
\hline $\begin{array}{c}\text { Science, Technology, } \\
\text { Engineering, and Math }\end{array}$ & 0 & 0 & 1 & 0 & 0 \\
\hline $\begin{array}{c}\text { Security and Emergency } \\
\text { Services }\end{array}$ & 0 & 13 & 3 & 4 & 2 \\
\hline Globalization & 0 & 0 & 0 & 0 & 0 \\
\hline SUBTOTAL & $\mathbf{2 9}$ & $\mathbf{4 1}$ & $\mathbf{2 4}$ & $\mathbf{2 0}$ & $\mathbf{2 1}$ \\
\hline
\end{tabular}

Section 6 - Research and Economic Development

TABLE 6A. Research and Development

R\&D Expenditures

\begin{tabular}{|l|l|l|l|l|}
\hline $2005-06$ & $2006-07$ & $2007-08$ & $2008-09$ & $2009-10$ \\
\hline
\end{tabular}

Federally Funded

Expenditures

(Thousand \$)

Total Expenditures

(Thousand \$)

Total R\&D Expenditures

Per Full-Time, Tenured,

Tenure-Earning Faculty

Member (\$)

Technology Transfer

Invention Disclosures

Total U.S. Patents Issued

Patents Issued Per 1,000

Full-Time, Tenure and

Tenure-Earning Faculty

Total Number of Licenses/

Reported at the USF System level

Options Executed

Total Licensing Income

Received (\$)

Total Number of Start-Up

Companies

Note: Awards and Expenditures are based on the National Science Foundation's annual Survey of R\&D

Expenditures at Universities and Colleges (data include Science \& Engineering and non-Science \&

Engineering awards). Technology Transfer data are based on the Association of University Technology

Managers Annual Licensing Survey.

USF Sarasota-Manatee 2011 Annual Report 